\title{
Collisional Activation of Protein Complexes: Picking Up the Pieces
}

\author{
Justin L. P. Benesch \\ Department of Chemistry, University of Cambridge, Cambridge, United Kingdom
}

\begin{abstract}
Mass spectrometry is fast becoming a vital approach not only for the identification and quantification of proteins, but also for the study of the noncovalent assemblies they form. Approaches for ionizing, transmitting, and detecting protein complexes intact in the mass spectrometer are now well established. The challenge has therefore shifted to developing and applying mass spectrometry approaches to elucidate the structure of such species. A crucial aspect to this goal is inducing their disassembly in the gas phase to mine information as to their composition and organization. Here the consequences of collisionally activating protein complexes are illustrated through ion mobility mass spectrometry measurements and discussed in the context of the current literature. Although a consensus view of the mechanism of dissociation is starting to emerge, it is also clear that a number of aspects remain unresolved. These outstanding questions and frontier challenges must be addressed if gas-phase dissociative approaches are to reach their full potential in the study of protein assemblies. (J Am Soc Mass Spectrom 2009, 20, 341-348) (C) 2009 Published by Elsevier Inc. on behalf of American Society for Mass Spectrometry
\end{abstract}

$\mathrm{P}$ otein assemblies were first analyzed intact in the gas phase by means of mass spectrometry (MS) at the beginning of the 1990s [1]. Since that time technological and methodological developments have continued apace [2-5], such that the MS of such large species is no longer merely a technical curiosity, but rather a bona fide approach for structural biologists [6]. The many advantages that MS possesses, including speed and sensitivity of analysis [7], have made it integral to the fields of proteomics and systems biology [8, 9]. The long-term challenge now is to extend the technology and methodology such that all higher levels of protein structure, from the secondary to the quinary [10], might be characterized rapidly and effectively by means of MS.

Such a revolution will require the addition to, and adaptation of, the current conventional MS-based proteomic strategy. A cornerstone of this is tandem MS, wherein ions of interest are subjected to gas-phase dissociation and the fragments analyzed to provide protein sequence, and thus identity, information [11]. Recently much effort has been made to perform analogous experiments on protein complexes, whereby they are dissociated in the mass spectrometer to provide mass information on their constituents [12]. Furthermore, some evidence suggests that, aside from just providing the identity of subunits, the gas-phase dissociation process may even reveal details as to how these

Address reprint requests to Dr. Justin L. P. Benesch, University of Cambridge, Department of Chemistry, Cambridge CB2 1EW, UK. E-mail: jlpb2@cam.ac.uk subunits are organized within the oligomer [13]. As such, the possibility has arisen that gas-phase dissociation coupled to MS might eventually allow the reverse engineering of protein assemblies.

Over the last few years a considerable body of literature has emerged regarding the mechanism of the gas-phase dissociation of protein assemblies. A variety of activation techniques have been used [14-18], but the most popular approach for the gas-phase dissociation of protein assemblies is currently collision-induced dissociation (CID). It is likely this is primarily attributable to its ease of implementation and its incorporation into the quadrupole time-of-flight $(\mathrm{Q}-\mathrm{ToF})$ instrument geometry currently favored in the study of protein assemblies [3]. Remarkably, despite the differences in activation techniques, the respective pathways of dissociation show a degree of overlap. As such, although the following discussion applies specifically to the CID of protein complexes, much of it applies to the various current activation methods.

CID relies on the activation arising from the collision of ions with neutral gas atoms or molecules [19]. Upon each collision with a gas molecule a small amount of an ion's energy is converted from kinetic into internal modes [20, 21]. In a typical CID experiment, large protein complexes will undergo thousands of such small heating events, over tens of microseconds [3]. Internal energy is therefore accumulated gradually, allowing time for its distribution over the many degrees of freedom as well as some dissipation to the environment. The general scheme of events as a protein complex is collisionally activated can be broken down into
(C) 2009 Published by Elsevier Inc. on behalf of American Society for Mass Spectrometry. 1044-0305/09/\$32.00

doi:10.1016/j.jasms.2008.11.014
Published online November 27, 2008 Received October 13, 2008 Revised November 19, 2008 Accepted November 19, 2008 
distinct steps, depending on the amount of internal energy available: cleaning, restructuring, unfolding, dissociating, and fragmenting.

This article illustrates these general features of the CID pathway through an ion mobility mass spectrometry (IM-MS) study of Acr1, a 197-kDa small heat shock protein from Mycobacterium tuberculosis composed of 12 identical subunits arranged as a hollow tetrahedron [22]. Recently, being able to perform IM-MS on mass spectrometers well suited to the study of protein assemblies has changed the landscape of the MS field [23]. Early work showed that the native topology of protein assemblies could be preserved in the gas phase and thus the door was opened toward probing of the conformational changes that these species undergo during activation [24]. IM-MS measurements of Acr1, at a range of activation energies presented here, are used as a gateway to discuss the literature. Finally, the gaps in current knowledge and future challenges for the CID of protein assemblies for the next few years are outlined and discussed.

\section{Collision-Induced Cleaning}

Figure 1 shows IM-MS spectra for Acr1 at three different low accelerating voltages into the collision cell, with corresponding projections of the MS and drift time dimensions. The MS projections (top) clearly show a single species in the region of $7000 \mathrm{~m} / \mathrm{z}$, corresponding to the dodecamer, although the spectra are not identical. At $8 \mathrm{~V}$, the minimum required to achieve ion transmission under these conditions, a mass of 197,438 $\mathrm{Da}$ is measured, with a peak width at a half-height of 39.3 Th for the $29+$ charge state. The corresponding values for $40 \mathrm{~V}$ (red) and $80 \mathrm{~V}$ (green) are, respectively, 197,070 Da and 35.1 Th and 196,703 Da and 21.3 Th. The mass expected from the sequence of Acr1 is 196,626 Da. This reflects a general feature of protein complexes in the gas phase- that their measured mass is higher than that expected by the sequences of their components alone [25-28]. Moreover, upon gentle activation both the peak width and positive deviation from the sequence mass decrease, which is attributed to the presence and, subsequently, the removal of residual solvent and salt bound to the protein complex [25-28].

The improvement in mass spectrum quality that this collision-induced cleaning enables can reveal features in the spectra not visible at lower activation conditions, thereby increasing the information content in the mass spectra [29]. Furthermore, the peak width and mass deviation are related such that, from the measured peak width, the contribution of adducts can be deconvoluted from the measured mass to enable more accurate mass determination of heterogeneous complexes [30]. As such, mass spectra of protein complexes are routinely acquired at the highest activation energies at which the complexes remain intact [31].

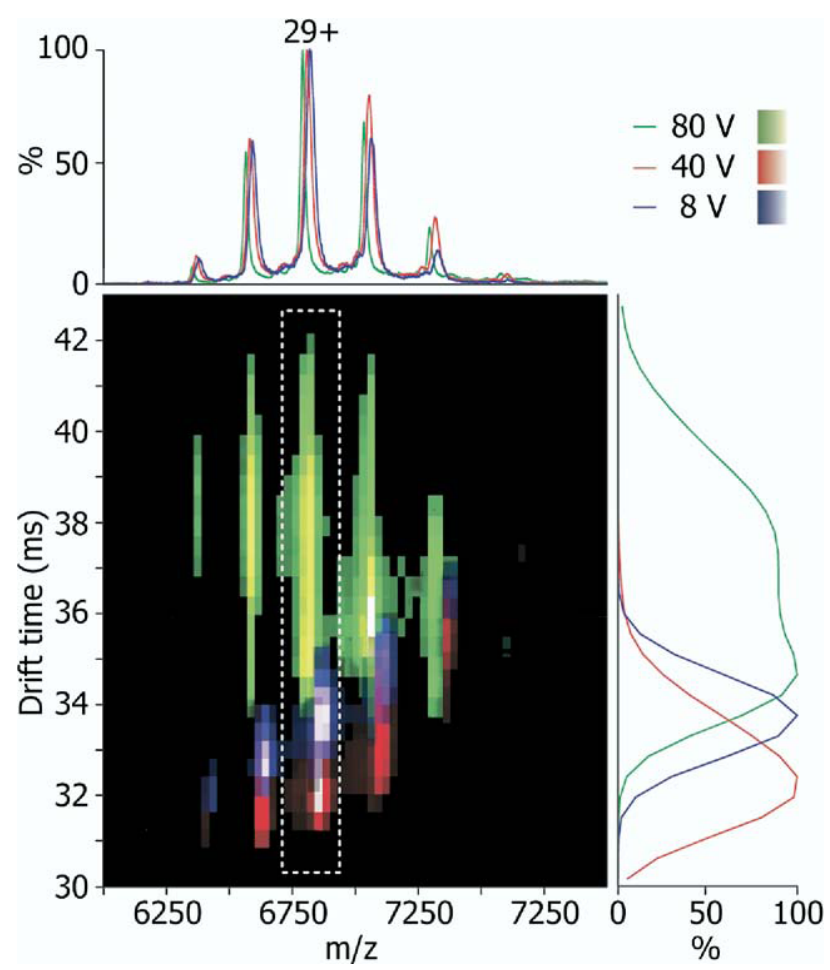

Figure 1. IM-MS spectra of Acr1 undergoing collisional activation. Accelerating voltages of $8 \mathrm{~V}$ (blue), $40 \mathrm{~V}$ (red), and $80 \mathrm{~V}$ (green) are shown. The MS dimension of these spectra, projected onto the top panel, shows charge-state series corresponding to a $197 \mathrm{kDa}$ dodecamer, in accordance with previous studies [22]. As the activation increases the MS dimension shows a decrease in both the peak width and $\mathrm{m} / \mathrm{z}$ of the charge states, attributed to removal of bound solvent and salt. The IM dimension for the modal $29+$ charge state is projected onto the right panel and intensities are normalized to $100 \%$. The drift time of the complex reduces from 8 to $40 \mathrm{~V}$, but increases from 40 to $80 \mathrm{~V}$. This is consistent with an oligomeric restructuring event, followed by an unfolding of one of the monomers in the complex.

\section{Collision-Induced Restructuring}

Concomitant with the changes observed in the MS dimension, changes in the drift time of Acr1 are also observed during this activation (Figure 1). At the lowest acceleration voltage the drift time of the $29+$ charge state occupies a narrow and symmetrical distribution centered on $33.8 \mathrm{~ms}$. At $40 \mathrm{~V}$ the distribution has broadened and skewed, with a maximum at the shorter drift time of $32.0 \mathrm{~ms}$. This indicates that some degree of restructuring of the complex occurs during gentle activation, resulting in a more compact form. A similar effect has been observed for the ring-shaped tryptophan RNA-binding attenuation protein, with the compact conformations consistent with structures in which the ring topology had collapsed into globular forms [24]. Acr1 similarly has a central cavity [22] and thus the reduction in drift time observed here at $40 \mathrm{~V}$ is likely the result of a collapse of the quaternary structure. In the case of the barrel-shaped GroEL, restructuring was also observed, but appeared to be attenuated in the presence of involatile buffer components [32]. Although the generality 
and mechanism of this apparent stabilization remain to be investigated, this suggests that solution additives could be used to stabilize protein complexes in the gas phase [33].

It appears therefore that the first structural change that protein complexes undergo during activation is one in which the overall complex arrangement becomes compromised, creating structures distorted from the solution form, but doing so without dissociation of the intact oligomers. This is likely to be a direct result of the removal of solvent [34], through the collision-induced cleaning process, which occurs at these same low activation energies (Figure 1). However, of the steps along the dissociation pathway, this collision-induced restructuring is by some distance the least well understood, in terms of both its nature and its extent. As such, it merits considerable attention in the coming years and quantitative IM-MS measurements are likely to contribute substantially to the understanding of this phenomenon. Although restructuring to forms other than collapsed cannot be discounted at this stage, it might be that the observation of this effect could prove diagnostic for assessing the presence of a cavity in protein assemblies. It is clear, however, that whereas the quality of the MS dimension is improved at $40 \mathrm{~V}$ relative to $8 \mathrm{~V}$, the IM dimension reveals the complex is further from the native solution form. In other words, the conditions necessary for high-quality mass spectra do not generally coincide with those for meaningful IM data on solution-phase structure [23].

\section{Collision-Induced Unfolding}

Additional changes in drift time are observed as the accelerating voltage is increased further. At $80 \mathrm{~V}$ the distribution has become multimodal and shifted to longer drift times (Figure 1). This effect has been observed for the activation of a number of protein assemblies [23, 35] and is consistent with a protein unfolding event $[36,37]$. The notion that monomer unfolding occurs during the activation of protein assemblies is well established. One of the first studies on the CID of protein assemblies speculated that dissociation might occur via the "unraveling" of a subunit, which is subsequently ejected [38]. Several pieces of evidence emerged in the following years consistent with this hypothesis. First, the charge states of the ejected monomer were very high [38], comparable with those for denatured monomers [39]; second, large entropy gains were observed in the dissociative transition state [15]; and, third, altering the flexibility of constituent subunits was found to alter the dissociation pathway $[40,41]$.

As such, apparently after the collision-induced restructuring of protein complexes, the next step involves a compromising of the intrasubunit interactions, resulting in the unfolding of the protein chains. A number of aspects of this process, however, remain somewhat controversial. How many subunits undergo unfolding?
Moreover, is their unfolding driven by the charge placed on the subunit(s) or does charge migrate to surfaces exposed during thermal unfolding?

Addressing the first question, detailed IM-MS measurements of the transthyretin tetramer were shown to be consistent with structures in which either one or more of the subunits were partially unfolded [35]. Crucially, although the two alternatives could not be separated, no species were measured that were larger than could be explained by the unfolding of only a single monomer [35]. Further support for unfolding being restricted primarily to a single monomer comes from the prediction that charge is apportioned between the unfolded monomer and the residual "stripped oligomer," according to fractional surface area [42] having been found to hold experimentally, using models in which the stripped oligomer is compact rather than unfolded [13]. Similarly an electrostatic model based on dissociative transition states composed of variably unfolded monomers, but folded stripped oligomers, accounted for the charge partitioning between components observed experimentally [43].

Recently, the role of charge in the actual unfolding process has also become clearer. Although a uniform spreading of charge over the surface of the protein assembly is the energetic "ideal," this is not attainable in practice because of the preferential localization of charges on nonuniformly spread basic sites [44]. Therefore gas-phase protein complexes are characterized not only by different charge states, but also by different distributions of the charges, coined charge isomers [45], over the protein structure. An important molecular dynamics study has shown that a monomer in a complex carrying more charge than that of the others undergoes structural relaxation and is more susceptible to thermal unfolding [44]. Therefore, as the internal energy of the system is increased upon collisional activation, unfolding of such coulombically compromised monomers occurs. Concomitantly, charge migrates to the surface area exposed by the unfolding process to maintain a surface charge density as uniform as possible [13,44]. Because the early events of protein unfolding are generally rapid and cooperative [46], and reversing this charge redistribution would be energetically very unfavorable, this puts the protein complex on an essentially irreversible path toward dissociation.

\section{Collision-Induced Dissociation}

Dissociation occurs when sufficient monomer unfolding has occurred and, concomitantly, sufficient charge has migrated to this monomer, that coulombic repulsion between components overcomes the intersubunit interactions holding the complex together. Figure 2a shows IM-MS spectra of Acr1 at accelerating voltages where dissociation begins to occur, with the corresponding projection of the MS and IM dimensions. Examining the MS dimension (top) reveals that at $80 \mathrm{~V}$ (green, as in Figure 1) the dodecamer is on the threshold 


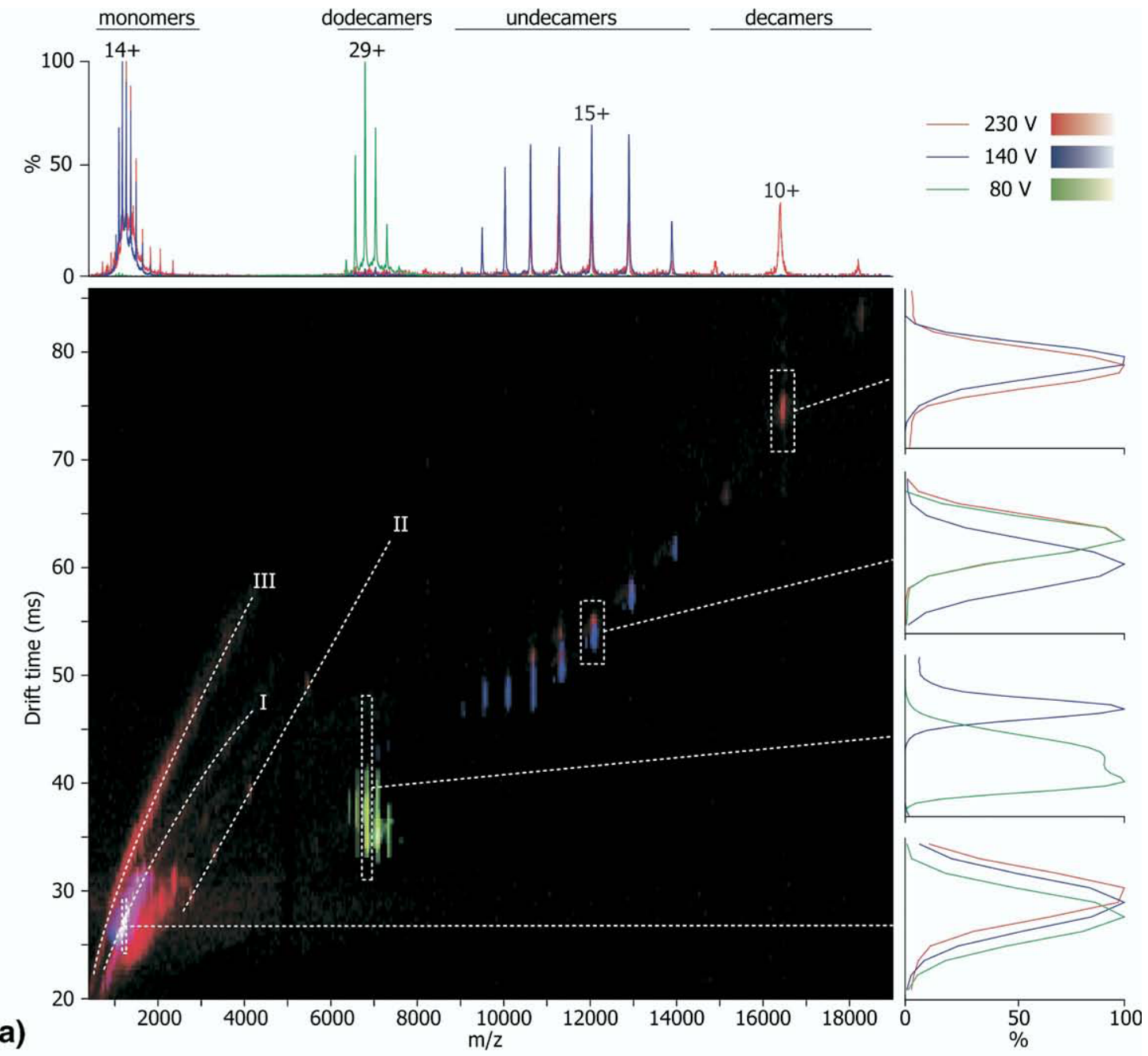

(a)

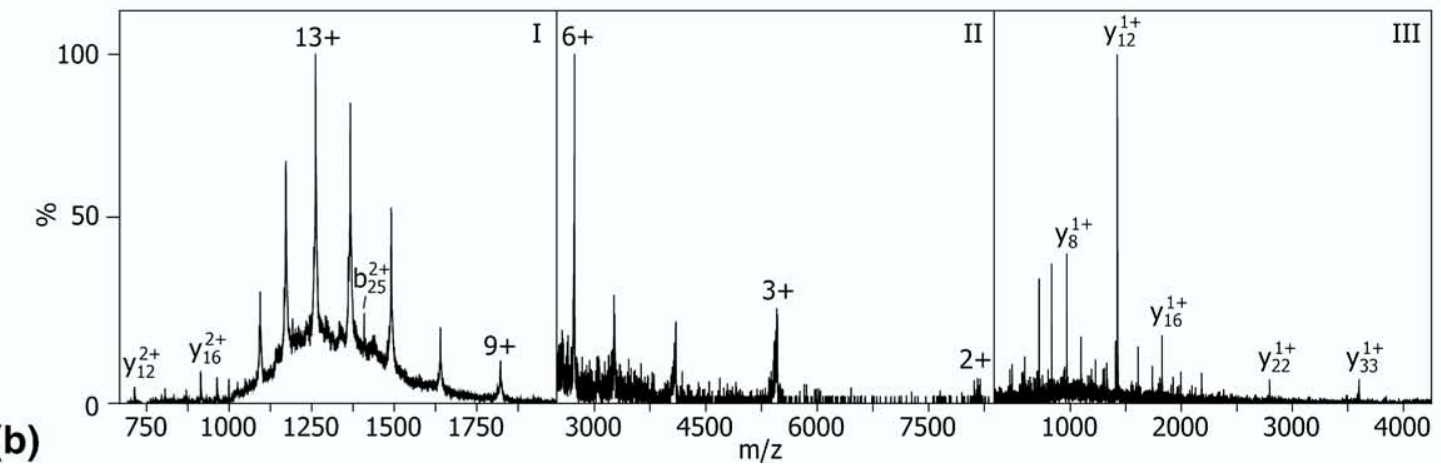

Figure 2. (a) IM-MS spectra of Acr1 undergoing CID. Accelerating voltages of $80 \mathrm{~V}$ (green), $140 \mathrm{~V}$ (blue), and $230 \mathrm{~V}$ (red) are shown. The MS dimension of these spectra is projected onto the top panel, with the range above $6000 \mathrm{~m} / \mathrm{z}$ magnified two- and 5-fold for 140 and $230 \mathrm{~V}$, respectively. Charge-state series corresponding to monomers, dodecamers, undecamers, and decamers can be observed, with more of the dissociation products appearing at higher accelerating voltages. Dissociation occurs via the loss of single monomers, in sequential fashion, from the dodecamer. The IM dimension for the 14+ monomers, $29+$ dodecamer, $15+$ undecamer, and $10+$ decamer is projected onto the right panel and intensities are normalized to $100 \%$. These correspond to drift time ranges of $24-29,31-48,51-57$, and $72-80 \mathrm{~ms}$, respectively. The stripped oligomers appear to undergo the same processes of restructuring and unfolding as observed for the dodecamers (Figure 1). As the accelerating voltage is raised, the drift time of the monomers increases, indicating further unfolding of the protein chains. A number of trends (I, II, and III) can be observed in the IM-MS spectrum at low $\mathrm{m} / \mathrm{z}$. (b) The extracted mass spectra reveal these trends to correspond primarily to monomers that are highly charged (I), to those that are charged to a lesser degree (II), and to singly charged peptide fragments (III). Comparison with the Acr1 sequence shows the fragments to be $b$ and $y$ type ions and shows the exciting possibility of being able to obtain "top-down" proteomics data directly from protein assemblies in the gas phase. 
of dissociation, with a very small population of monomer and undecamer visible at low and high $\mathrm{m} / \mathrm{z}$, respectively. At $140 \mathrm{~V}$ (blue) considerably more of these complementary dissociation products are observed, with the abundance of dodecamer accordingly much lower. As the accelerating voltage is increased still further $(230 \mathrm{~V}$, red) decamer is observed around $18,000 \mathrm{~m} / \mathrm{z}$.

A number of observations can be made from the MS dimension. First, dissociation of the Acr1 dodecamer occurs via the loss of a monomer to form an undecamer. This might at first glance be somewhat surprising because electron microscopy analysis has shown Acr1 to be composed of dimeric building blocks [22]. However, the dissociation of large protein oligomers in the gas phase through the loss of single subunits to form singly stripped oligomers appears to be a general phenomenon [13], although some exceptions to this rule have been reported $[47,48]$, the implications of which are discussed in more detail in the following text. Second, because decamers are observed at the highest acceleration voltage, more than one monomer can be removed from Acr1. This loss of multiple subunits is a sequential process [13]-i.e., the Acr1 undecamers formed during the first dissociation step can themselves undergo CID. This process can occur repeatedly, limited by the absolute number of charges and activation energy available [49]. Indeed, in favorable cases as many as seven successive dissociation steps have been observed [50].

Third, from the charge-state distributions of the momomers (modal 14+) and undecamers (modal 15+) formed from dissociation of the dodecamer (modal $29+$ ) we can see that charge is conserved during CID and distributed approximately equally between the products. This even distribution of charge between products is the energetically favored charge configuration and is generally adopted [44]. In some cases, however, a monomer cannot unfold sufficiently to attain a surface area equal to that of the stripped oligomer, in which case the charge is distributed in accordance with the attained ratio of surface areas [13, 44]. This apportioning of charge governed by surface area between two products that are vastly different in mass leads to considerably different $m / z$ ratios for the monomers and stripped oligomers. A beneficial aspect of this is that removal of the highly charged monomers results in an effective charge reduction of the oligomers [12]. Charge reduction of electrosprayed species is highly useful in that it facilitates the deconvolution of multicomponent mixtures [51]. As such, a principal utility of CID is to exploit the increased separation between stripped oligomer peaks to deconvolve species not intelligible in the MS spectra [52].

A number of observations can be also made from the IM dimension (Figure 2a, right). At $80 \mathrm{~V}$, at the threshold of dissociation, the dodecamers occupy a distribution characteristic of an oligomer with a partially unfolded monomer (see previous section). The small number of dodecamers remaining at $140 \mathrm{~V}$ are even larger, indicating that, although the majority of the species have already dissociated, those that remained intact at this voltage could absorb more energy by further unfolding. This highlights the necessity of considering the dissociating oligomers as an ensemble of structures [44]. The undecamers also undergo changes in their drift-time profile as a function of acceleration voltage. From 80 to $140 \mathrm{~V}$ the $15+$ charge state becomes smaller, then from 140 to $230 \mathrm{~V}$, larger again. The profiles of the decamer $10+$ charge state for 140 and 230 $\mathrm{V}$ also show the beginnings of similar behavior, with the drift time at $230 \mathrm{~V}$ being slightly shorter than that at $140 \mathrm{~V}$. This is analogous to the collision-induced restructuring and unfolding observed for the dodecamers (see previous text), reinforcing the view that subsequent dissociation steps follow the same pathway as that of the initial one $[13,44]$. It must be stressed that these IM measurements are only qualitative, however, and quantitative measurements with the appropriate modeling need to be conducted to reveal the full details of these structural transitions.

\section{Collision-Induced Fragmentation}

In Figure 2a it is also apparent that differences can be observed at low $\mathrm{m} / \mathrm{z}$ as the voltage is increased from 140 to $230 \mathrm{~V}$. Examination of the $14+$ monomer charge state shows that it appears to become slightly larger as accelerating voltage increases. This increase in size could be indicative of gas-phase protein unfolding [36, 37] and can be explained by the monomer ejected by CID being activated after ejection by subsequent collisions, thereby unfolding further.

Furthermore, at $230 \mathrm{~V}$ a number of "trend lines" can be seen in the IM-MS spectrum and these are extracted into mass spectra in Figure 2b. Trend I shows highly charged monomers and is the only trend also visible at $140 \mathrm{~V}$, indicating this corresponds to monomers released in the first dissociation step. Trend II also shows monomers, but these are charged to a lesser degree and because they appear only at $230 \mathrm{~V}$ are likely to arise from the second dissociation step. Trend III, however, does not display any features corresponding to monomers, but rather corresponds to singly charged peptides. In fact 26 peptides can be identified as $b$ - and $y$-type fragments of the Acr1 protein chain. Closer inspection of Trend I also allows the identification of a further 7 peptide ions, in this case multiply charged. Strikingly, the vast majority of fragments observed were $y$ type (30 y versus $3 b$ ). Because the folding state of a protein appears to influence which cleavages are observed, with more cleavages at unfolded regions [53], this may suggest that the C-terminal region of the ejected Acr1 monomers is more unfolded than the N-terminus. This is only one of the potential explanations, but interestingly matches the notion that the C-termini of the small heat shock proteins are more flexible and act as "keys" to subunit unfolding [54, 55]. Although what information can be obtained from 
quantifying the relative populations of different peptide ions remains to be investigated, clearly collisional activation of protein assemblies leads not only to their dissociation, but also ultimately to fragmentation of the constituent protein chains. This essentially amounts to an extension of traditional "top-down" proteomics [56], allowing information as to the complex, subunit, and sequence to be gleaned in a single experiment [49].

\section{Frontiers in Gas-Phase Activation of Protein Complexes}

The case study of Acr1 here has provided a glimpse of some of the information obtainable through gas-phase dissociation. Commercial mass spectrometers suitable for not only the ionization and transmission of large protein complexes, but also their selective CID, have in recent years become considerably more available, with a number of academic labs worldwide now enjoying the technology. The use of collisional activation is therefore becoming widespread and is proving essentially indispensible in the study of protein complexes by means of MS. Most studies to date use CID for the purposes of mass measurement, either to confirm the apparent mass obtained in the MS spectrum or to determine the mass of a particular component within a heterogeneous mixture [12].

Although the application of dissociative approaches to determining oligomeric stoichiometry is now well established, the use of CID to obtain details with respect to oligomeric organization is in its infancy. A growing base of evidence suggests that the ease of dissociation of the different constituent subunits might indicate whether they are located on the periphery or in the core of the complexes [57-61]. Validation of this hypothesis would allow confident inferences as to oligomeric topology from CID results, but a crucial twofold question remains to be answered: "In a heteromeric complex, what governs which subunits are ejected during CID and how does this relate to solution-phase topology?"

To answer this question likely most steps on the pathway of collisional activation delineated here need to be considered. How does a certain complex restructure? Which subunits are most likely to carry excess charge and thus unfold? Are all unfolding pathways capable of attaining a dissociative transition state or are there steric constraints? Which of these transition states is reached first? These are all important considerations, and likely inherent properties-such as the location of basic residues, the length of the protein chains, the kinetic stability of different subunits relative to unfolding, and the strength of intersubunit interactions-all contribute. One of the dominant challenges for the application of CID to structural biology over the next few years is to be able to parse out the contributions of complex topology from these other factors.

A related issue concerns homomeric complexes, such as Acr1 here, and whether clues as to suboligomeric organization might be obtained, such that, for example, a hexamer might be shown to be a dimer of trimers rather than a trimer of dimers [62]. There is evidence that CID can be used to demonstrate relative differences from careful examination of the energetics of dissociation [13, 63], although this approach is not currently applicable to a priori determination of substructure. Ideally, the dissociation products would directly represent the units of organization, rather than necessarily monomers and stripped oligomers, e.g., if for Acr1, which is composed of noncovalently bound dimers [22], CID of the dodecamer showed the ejection of dimers. Indeed, one of the major unanswered questions in this field is why, in the dissociative transition state, the contacts broken are between unfolded monomer and the remaining complex, even in cases where that unfolded monomer is part of a subcomplex with independent stability within the oligomer: in the context of Acr1, why does the unfolded monomer not take its dimer partner with it during dissociation?

A couple of studies have shown exceptions to the exclusive loss of monomers $[47,48]$, but it is unclear at this time why precisely they should behave differently. In the case of 2-keto-3-deoxyarabinonate, a tetramer observed to dissociate into dimers, the authors suggested through analysis of the crystal structure that the nature of the intersubunit contacts were responsible [48]. Contrastingly, the subunits of brown snake venom are conformationally very restricted because of many intramolecular disulfide bonds and it was suggested that this inability to unfold easily may have been responsible [47]. This is interesting in the context of previous work that showed that dissociation of dimers was more "symmetric" when the subunits were also crosslinked intramolecularly [41]. A priority in the study of CID of protein complexes is to identify more species that undergo such "anomalous" CID and to examine them in detail. Hopefully, it might thereafter become possible to delineate experimental conditions where dissociation reveals the suboligomeric organization.

It might be that accessing alternative dissociation pathways-ones that clearly reveal solution-phase substructure-might arise through the use of activation strategies other than CID. Black-body infrared radiative dissociation [15], electron-capture dissociation [17], infrared multiphoton dissociation [14, 16], and surface-induced dissociation $[18,64]$ have all been applied to protein assemblies. Of these, the latter is notable for the large amount of energy deposited into the protein complexes in a very short time, resulting in more extensive dissociation than that in CID [65]. Furthermore, small multimeric complexes were shown to dissociate into equally charged monomeric parts, rather than monomers and stripped oligomers $[64,65]$. These differences are rationalized in terms of the collisions being more energetic in the center-of-mass frame and the speed of energy deposition being far greater [65]. Efforts have been made to bring these differences to CID, both by using heavier target gases [66] and by 
increasing the range of acceleration voltages in the collision cell $[49,67]$.

One consequence of collisional activation with higher energies is the appearance of peptide fragments arising from fragmentation of the ejected monomers [49]. The ability to perform "top-down" experiments on protein assemblies-simultaneously obtaining information on the mass of the complex, the mass of the subunits, and the sequence all in a single experimentis hugely attractive. This approach is still in its infancy, however, and warrants considerable further investigation.

A frontier challenge in biological science is to bridge the gap between structural biology and proteomics, making the determination of protein complex structure routine [68], and MS approaches are likely to play a considerable part in this effort [69-71]. Although the technologies and methodologies still require some refinement, clearly gas-phase dissociation has the potential to be as integral to analyzing such species as it is for identifying protein chains. As such, the MS of protein complexes might ultimately reveal enough information in a single rapid experiment to fully characterize the structure of an unknown protein complex.

\section{Experimental}

Acr1 was expressed in E. coli, purified, and prepared for analysis as described previously [22]. IM-MS measurements were obtained on a Synapt HDMS (Waters, Milford, MA, USA) [72], equipped with a 32K quadrupole and set up for the analysis of protein complexes [23]. Nanoelectrospray spectra were obtained in positive-ion mode using a previously described protocol [31].

The following voltages were used: capillary, $1.6 \mathrm{kV}$; sample cone, $10 \mathrm{~V}$; "transfer collision energy," $10 \mathrm{~V}$; injection into the T-wave cell, $30 \mathrm{~V}$; and a $0-$ to $30-\mathrm{V}$ wave-height ramp in the drift cell. The "trap collision energy" was varied to effect activation and is quoted as "accelerating voltage" in the main text. The gases used were nitrogen and argon in the T-wave IM separator and collision cells, respectively, at room temperature $\left(22^{\circ} \mathrm{C}\right)$. The pressures in the various stages were the following: backing, 4.8 mbar; trap, $7.2 \times 10^{-2}$ mbar; IMS, $6.1 \times 10^{-1}$ mbar; and ToF, $2.2 \times 10^{-6}$ mbar. At this pressure in the trap the ions are essentially thermalized before their injection into the IMS cell [3].

Data were processed using Masslynx software and the MS dimension was calibrated externally. All spectra are shown here with minimal smoothing (none in the case of the IM-MS 3D spectra) and no background subtraction. The 3D IM-MS spectra in Figure 1 have linear intensity scales and those in Figure 2 have square-root scales.

\section{Acknowledgments}

The author thanks Christopher Kennaway and Nicholas Keep (Birkbeck College, London) for providing Acr1; Alan Sandercock, Brandon Ruotolo, and Matthew Bush (University of Cambridge) for critical review; Carol Robinson (University of Cambridge) for support; and the Royal Society for funding.

\section{References}

1. Loo, J. A. Studying Noncovalent Protein Complexes by Electrospray Ionization Mass Spectrometry. Mass Spectrom. Rev. 1997, 16, 1-23.

2. Ashcroft, A. E. Recent Developments in Electrospray Ionisation Mass Spectrometry: Noncovalently Bound Protein Complexes. Nat. Prod. Rep. 2005, 22, 452-464.

3. Benesch, J. L. P.; Ruotolo, B. T.; Simmons, D. A.; Robinson, C. V. Protein Complexes in the Gas Phase: Technology for Structural Genomics and Proteomics. Chem. Rev, 2007, 107, 3544-3567.

4. Heck, A. J. R.; van den Heuvel, R. H. H. Investigation of Intact Protein Complexes by Mass Spectrometry. Mass Spectrom. Rev. 2004, 23, 368 389.

5. Wyttenbach, T.; Bowers, M. T. Intermolecular Interactions in Biomolecular Systems Examined by Mass Spectrometry. Annu. Rev. Phys. Chem. 2007, 58, 511-533.

6. Sharon, M.; Robinson, C. V. The Role of Mass Spectrometry in Structure Elucidation of Dynamic Protein Complexes. Annu. Rev. Biochem. 2007, 76, 167-193.

7. McLafferty, F. W.; Fridriksson, E. K.; Horn, D. M.; Lewis, M. A.; Zubarev, R. A. Techview: Biochemistry. Biomolecule Mass Spectrometry. Science 1999, 284, 1289-1290.

8. Aebersold, R.; Mann, M. Mass Spectrometry-based Proteomics. Nature 2003, 422, 198-207.

9. Cravatt, B. F.; Simon, G. M.; Yates, J. R., 3rd. The Biological Impact of Mass Spectrometry-based Proteomics. Nature 2007, 450, 991-1000.

10. McConkey, E. H. Molecular Evolution, Intracellular Organization, and the Quinary Structure of Proteins. Proc. Natl. Acad. Sci. U. S. A. 1982, 79, 3236-3240.

11. Domon, B.; Aebersold, R. Mass Spectrometry and Protein Analysis. Science 2006, 312, 212-217.

12. Benesch, J. L. P.; Robinson, C. V. Mass Spectrometry of Macromolecular Assemblies: Preservation and Dissociation. Curr. Opin. Struct. Biol. 2006 16, 245-251.

13. Benesch, J. L. P.; Aquilina, J. A.; Ruotolo, B. T.; Sobott, F.; Robinson, C. V. Tandem Mass Spectrometry Reveals the Quaternary Organization of Macromolecular Assemblies. Chem. Biol. 2006, 13, 597-605.

14. El-Faramawy, A.; Guo, Y.; Verkerk, U.; Thomson, B. A.; Siu, M. Evaluation of IR Multi Photon Dissociation as a Method for High Mass Protein Clean Up. In 56th ASMS Conference on Mass Spectrometry, Denver, CO, 2008.

15. Felitsyn, N.; Kitova, E. N.; Klassen, J. S. Thermal Decomposition of a Gaseous Multiprotein Complex Studied by Blackbody Infrared Radiative Dissociation. Investigating the Origin of the Asymmetric Dissociation Behavior. Anal. Chem. 2001, 73, 4647-4661.

16. Geels, R. B.; Calmat, S.; Heck, A. J.; van der Vies, S. M.; Heeren, R. M. Thermal Activation of the Co-Chaperonins GroES and gp31 Probed by Mass Spectrometry. Rapid Commun. Mass Spectrom. 2008, 22, 3633-3641.

17. Geels, R. B.; van der Vies, S. M.; Heck, A. J.; Heeren, R. M. Electron Capture Dissociation as Structural Probe for Noncovalent Gas-Phase Protein Assemblies. Anal. Chem, 2006, 78, 7191-7196.

18. Jones, C. M.; Beardsley, R. L.; Galhena, A. S.; Dagan, S.; Cheng, G.; Wysocki, V. H. Symmetrical Gas-Phase Dissociation of Noncovalent Protein Complexes via Surface Collisions. J. Am. Chem. Soc. 2006, 128, 15044-15045.

19. Jennings, K. R. The Changing Impact of the Collision-induced Decomposition of Ions on Mass Spectrometry. Int. J. Mass Spectrom. 2000, 200 $479-493$.

20. Shukla, A. K.; Futrell, J. H. Tandem Mass Spectrometry: Dissociation of Ions by Collisional Activation. I. Mass Spectrom 2000, 35, 1069-1090.

21. Sleno, L.; Volmer, D. A. Ion Activation Methods for Tandem Mass Spectrometry. J. Mass Spectrom. 2004, 39, 1091-1112.

22. Kennaway, C. K.; Benesch, J. L. P.; Gohlke, U.; Wang, L.; Robinson, C. V.; Orlova, E. V.; Saibil, H. R.; Keep, N. H. Dodecameric Structure of the Small Heat Shock Protein Acr1 from Mycobacterium Tuberculosis. J. Biol. Chem. 2005, 280, 33419-33425.

23. Ruotolo, B. T.; Benesch, J. L. P.; Sandercock, A. M.; Hyung, S. J.; Robinson, C. V. Ion Mobility-Mass Spectrometry Analysis of Large Protein Complexes. Nat. Protoc. 2008, 3, 1139-1152.

24. Ruotolo, B. T.; Giles, K.; Campuzano, I.; Sandercock, A. M.; Bateman, R. H.; Robinson, C. V. Evidence for Macromolecular Protein Rings in the Absence of Bulk Water. Science 2005, 310, 1658-1661.

25. Senko, M. W.; Speir, J. P.; McLafferty, F. W. Collisional Activation of Large Multiple Charged Ions Using Fourier Transform Mass Spectrometry. Anal. Chem. 1994, 66, 2801-2808.

26. Smith, R. D.; Loo, J. A.; Barinaga, C. J.; Edmonds, C. G.; Udseth, H. R. Collisional Activation and Collision-Activated Dissociation of Large Multiply Charged Polypeptide and Proteins Produced by Electrospray Ionization. J. Am. Soc. Mass Spectrom. 1990, 1, 53-65.

27. Sobott, F.; Robinson, C. V. Characterising Electrosprayed Biomolecules Using Tandem-MS: The Noncovalent GroEL Chaperonin Assembly. Int. J. Mass Spectrom. 2004, 236, 25-32.

28. Tolić, L. P.; Bruce, J. E.; Lei, Q. P.; Anderson, G. A.; Smith, R. D. In-Trap Cleanup of Proteins from Electrospray Ionization Using Soft Sustained 
Off-resonance Irradiation with Fourier Transform Ion Cyclotron Resonance Mass Spectrometry. Anal. Chem, 1998, 70, 405-408.

29. Ilag, L. L.; Westblade, L. F.; Deshayes, C.; Kolb, A.; Busby, S. J.; Robinson, C. V. Mass Spectrometry of Escherichia coli RNA Polymerase: Interactions of the Core Enzyme with Sigma70 and Rsd Protein. Structure 2004, 12, 269-275.

30. McKay, A. R.; Ruotolo, B. T.; Ilag, L. L.; Robinson, C. V. Mass Measurements of Increased Accuracy Resolve Heterogeneous Populations of Intact Ribosomes. J. Am. Chem. Soc. 2006, 128, 11433-11442.

31. Hernández, H.; Robinson, C. V. Determining the Stoichiometry and Interactions of Macromolecular Assemblies from Mass Spectrometry. Nat. Protoc. 2007, 2, 715-726.

32. Freeke, J.; Robinson, C. V.; Ruotolo, B. T. Residual counter ions can stabilise a large protein complex in the gas phase. Int. J. Mass Spectrom. $\mathbf{2 0 0 8}$, in preparation.

33. Sun, J.; Kitova, E. N.; Klassen, J. S. Method for Stabilizing ProteinLigand Complexes in Nanoelectrospray Ionization Mass Spectrometry. Anal. Chem. 2007, 79, 416-425.

34. Steinberg, M. Z.; Elber, R.; McLafferty, F. W.; Gerber, R. B.; Breuker, K. Early Structural Evolution of Native Cytochrome c after Solvent Removal. ChemBioChem 2008, 9, 2417-2423.

35. Ruotolo, B. T.; Hyung, S. J.; Robinson, P. M.; Giles, K.; Bateman, R. H. Robinson, C. V. Ion Mobility-Mass Spectrometry Reveals Long-lived, Unfolded Intermediates in the Dissociation of Protein Complexes. Angew. Chem. Int. Ed. Engl. 2007, 46, 8001-8004.

36. Clemmer, D. E.; Hudgins, R. R.; Jarrold, M. F. Naked Protein Conformations: Cytochrome $\mathrm{c}$ in the Gas Phase. J. Am. Chem. Soc. 1995, 117, 10141-10142.

37. Clemmer, D. E., Jarrold, M. F. Ion Mobility Measurements and Their Applications to Clusters and Biomolecules. J. Mass Spectrom. 1997, 32, 577-598.

38. Light-Wahl, K. J: Schwartz, B. L.; Smith, R. D. Observation of the Noncovalent Quaternary Associations of Proteins be Electrospray Ionization Mass Spectrometry. J. Am. Chem. Soc. 1994, 116, 5271-5278.

39. Chowdhury, S. K.; Katta, V.; Chait, B. T. Probing ConformationalChanges in Proteins By Mass-Spectrometry. J. Am. Chem. Soc. 1990, 112, 9012-9013.

40. Jurchen, J. C.; Garcia, D. E.; Williams, E. R. Further Studies on the Origins of Asymmetric Charge Partitioning in Protein Homodimers. J. Am. Soc. Mass Spectrom. 2004, 15, 1408-1415.

41. Jurchen, J. C.; Williams, E. R. Origin of Asymmetric Charge Partitioning in the Dissociation of Gas-Phase Protein Homodimers. J. Am. Chem. Soc. 2003, 125, 2817-2826.

42. Csiszar, S.; Thachuk, M. Using Ellipsoids to Model Charge Distributions in Gas Phase Protein Complex Ion Dissociation. Can. J. Chem. 2004, 82, $1736-1744$.

43. Sinelnikov, I. Kitova, E. N.; Klassen, J. S. Influence of Coulombic Repulsion on the Dissociation Pathways and Energetics of Multiprotein Complexes in the Gas Phase. J. Am. Soc. Mass Spectrom. 2007, 18, 617631.

44. Wanasundara, S. N.; Thachuk, M. Theoretical Investigations of the Dissociation of Charged Protein Complexes in the Gas Phase. J. Am. Soc. Mass Spectrom. 2007, 18, 2242-2253.

45. Felitsyn, N.; Kitova, E. N.; Klassen, J. S. Thermal Dissociation of the Protein Homodimer Ecotin in the Gas Phase. J. Am. Soc. Mass Spectrom. 2002, 13, 1432-1442.

46. Daggett, V.; Levitt, M. Protein Unfolding Pathways Explored through Molecular Dynamics Simulations. J. Mol. Biol. 1993, 232, 600-619.

47. Aquilina, J. A. The Major Toxin from the Australian Common Brown Snake Is a Hexamer with Unusual Gas-Phase Dissociation Properties. Proteins 2008, doi:10.1002/prot.22259.

48. van den Heuvel, R. H.; van Duijn, E.; Mazon, H.; Synowsky, S. A. Lorenzen, K.; Versluis, C.; Brouns, S. J.; Langridge, D.; van der Oost, J.; Hoyes, J.; Heck, A. J. Improving the Performance of a Quadrupole Time-of-Flight Instrument for Macromolecular Mass Spectrometry. Anal. Chem. 2006, 78, 7473-7483.

49. Benesch, J. L. P.; Ruotolo, B. T.; Sobott, F.; Wildgoose, J.; Gilbert, A. Bateman, R.; Robinson, C. V. A Q-ToF Mass Spectrometer Modified for Higher-Energy Dissociation Reduces Protein Assemblies to Peptide Fragments. Anal. Chem. 2008, in press.

50. Uetrecht, C.; Versluis, C.; Watts, N. R.; Roos, W. H.; Wuite, G. J.; Wingfield, P. T. Steven, A. C. Heck, A. J. High-Resolution Mass Spectrometry of Viral Assemblies: Molecular Composition and Stability of Dimorphic Hepatitis B Virus Capsids. Proc. Natl. Acad. Sci. U. S. A. 2008, 105, 9216-9220
51. Scalf, M.; Westphall, M. S.; Krause, J.; Kaufman, S. L.; Smith, L. M. Controlling Charge States of Large Ions. Science 1999, 283, 194-197.

52. Aquilina, J. A.; Benesch, J. L. P.; Bateman, O. A.; Slingsby, C.; Robinson, C. V. Polydispersity of a Mammalian Chaperone: Mass Spectrometry Reveals the Population of Oligomers in alphaB-Crystallin. Proc. Natl. Acad. Sci. U. S. A. 2003, 100, 10611-10616.

53. Han, X.; Jin, M.; Breuker, K.; McLafferty, F. W. Extending Top-Down Mass Spectrometry to Proteins with Masses Greater Than 200 Kilodaltons. Science 2006, 314, 109-112.

54. Aquilina, J. A.; Benesch, J. L. P.; Ding, L. L.; Yaron, O.; Horwitz, J.; Robinson, C. V. Subunit Exchange of Polydisperse Proteins: Mass Spectrometry Reveals Consequences of alphaA-Crystallin Truncation. J. Biol. Chem. 2005, 280, 14485-14491.

55. Carver, J. A.; Aquilina, J. A.; Truscott, R. J.; Ralston, G. B. Identification by $1 \mathrm{H}$ NMR Spectroscopy of Flexible C-Terminal Extensions in Bovine Lens alpha-Crystallin. FEBS Lett. 1992, 311, 143-149.

56. Kelleher, N. L.; Lin, H. Y.; Valaskovic, G. A.; Aaserud, D. J.; Fridriksson, E. K.; McLafferty, F. W. Top Down versus Bottom Up Protein Characterization by Tandem High-Resolution Mass Spectrometry. J. Am. Chem. Soc. 1999, 121, 806-812.

57. Chernushevich, I. V.; Thomson, B. A. Collisional Cooling of Large Ions in Electrospray Mass Spectrometry. Anal. Chem. 2004, 76, 1754-1760.

58. Hernández, H.; Dziembowski, A.; Taverner, T.; Seraphin, B.; Robinson, C. V. Subunit Architecture of Multimeric Complexes Isolated Directly from Cells. EMBO Rep. 2006, 7, 605-610.

59. Ilag, L. L.; Videler, H.; McKay, A. R.; Sobott, F.; Fucini, P.; Nierhaus, K. H.; Robinson, C. V. Heptameric (L12)6/L10 Rather Than Canonical Pentameric Complexes Are Found by Tandem MS of Intact Ribosomes from Thermophilic Bacteria. Proc. Natl. Acad. Sci. U. S. A. 2005, 102, 8192-8197.

60. Sharon, M.; Witt, S.; Felderer, K.; Rockel, B.; Baumeister, W.; Robinson, C. V. 20S Proteasomes Have the Potential to Keep Substrates in Store for Continual Degradation. J. Biol. Chem. 2006, 281, 9569-9575.

61. van Duijn, E.; Simmons, D. A.; van den Heuvel, R. H.; Bakkes, P. J.; van Heerikhuizen, H.; Heeren, R. M.; Robinson, C. V.; van der Vies, S. M. Heck, A. J. R. Tandem Mass Spectrometry of Intact GroEL-Substrate Complexes Reveals Substrate-specific Conformational Changes in the trans Ring. J. Am. Chem. Soc. 2006, 128, 4694-4702.

62. Levy, E. D.; Boeri Erba, E.; Robinson, C. V.; Teichmann, S. A. Assembly Reflects Evolution of Protein Complexes. Nature 2008, 453, 1262-1265.

63. Benesch, J. L. P.; Ayoub, M.; Robinson, C. V.; Aquilina, J. A. Small Heat Shock Protein Activity Is Regulated by Variable Oligomeric Substructure. J. Biol. Chem. 2008, 283, 28513-28517.

64. Wysocki, V. H.; Joyce, K. E.; Jones, C. M.; Beardsley, R. L. SurfaceInduced Dissociation of Small Molecules, Peptides, and Non-Covalen Protein Complexes. J. Am. Soc. Mass Spectrom. 2008, 19, 190-208.

65. Wysocki, V. H.; Jones, C. M.; Galhena, A. S.; Blackwell, A. E. SurfaceInduced Dissociation Shows Potential to Be More Informative Than Collision-Induced Dissociation for Structural Studies of Large Systems. J. Am. Soc. Mass Spectrom. 2008, 19, 903-913.

66. Lorenzen, K.; Versluis, C.; van Duijn, E.; van den Heuvel, R. H.; Heck, A. J. R. Optimizing Macromolecular Tandem Mass Spectrometry of Large Non-Covalent Complexes Using Heavy Collision Gases. Int. J. Mass Spectrom. 2007, 268, 198-206.

67. Ackloo, S.; Chernushevich, I. V.; Loboda, A.; Haufler, R. E.; Thomson, B. A. Accessing Collision Energies of up to $500 \mathrm{~V}$ per Charge on a QqToF Instrument: Structure Characterization of Fullerenes and Large Peptides. In Proceedings of the 56th ASMS Conference on Mass Spectrometry, Denver, CO, 2008.

68. Sali, A.; Glaeser, R.; Earnest, T.; Baumeister, W. From Words to Literature in Structural Proteomics. Nature 2003, 422, 216-225.

69. Gingras, A. C.; Gstaiger, M.; Raught, B.; Aebersold, R. Analysis of Protein Complexes Using Mass Spectrometry. Nat. Rev. Mol. Cell. Biol. 2007, 8, 645-654.

70. Heck, A. J. Native Mass Spectrometry: A Bridge between Interactomics and Structural Biology. Nat. Methods 2008, 5, 927-933.

71. Robinson, C. V.; Sali, A.; Baumeister, W. The Molecular Sociology of the Cell. Nature 2007, 450, 973-982.

72. Pringle, S. D.; Giles, K.; Wildgoose, J. L.; Williams, J. P.; Slade, S. E.; Thalassinos, K.; Bateman, R. H.; Bowers, M. T.; Scrivens, J. H. An Investigation of the Mobility Separation of Some Peptide and Protein Ions Using a New Hybrid Quadrupole/Travelling Wave IMS/oa-ToF Instrument. Int. J. Mass Spectrom. 2007, 261, 1-12. 